\title{
Mutual Coupling Reduction in Microstrip Antennas using Defected Ground Structure
}

\author{
Nguyen Ngoc Lan \\ Faculty of Electronics and Telecommunications, Saigon University, Viet Nam \\ Correspondence: Nguyen Ngoc Lan, ngoclannguyen@sgu.edu.vn \\ Communication: received 3 February 2020, revised 27 April 2020, accepted 19 May 2020 \\ Online publication: 10 June 2020, Digital Object Identifier: 10.21553/rev-jec.249 \\ The associate editor coordinating the review of this article and recommending it for publication was Prof. Vo Nguyen Quoc Bao.
}

\begin{abstract}
A Multiple Input Multiple Output (MIMO) antenna with high isolation is proposed in this paper. The proposed antenna includes two sets of four elements $(2 \times 2)$ and it is yielded at the central frequency of $5.5 \mathrm{GHz}$ for Wireless Local Area Network (WLAN) applications. Based on RT5880 with height of $1.575 \mathrm{~mm}$, the overall size of MIMO antenna is $140 \times 76 \times 1.575 \mathrm{~mm}^{3}$. To get high isolation between antenna elements, a Defected Ground Structure (DGS) is integrated on ground plane. Besides, the MIMO antenna witnesses a large bandwidth of $9.1 \%$ and an efficiency of $90 \%$ while the pick gain is $8.5 \mathrm{dBi}$. The measurement results are compared to simulation ones to verify the performance of the proposed antenna.
\end{abstract}

Keywords- Array antenna, MIMO antenna, defected ground structure, dgs, mutual coupling.

\section{INTRODUCTION}

Nowadays, microstrip patch antennas are commonly used in modern wireless systems thanks to their advantages, for example: light weight, easy fabrication and integration into PCB circuits. However, there are some performance limitations in them such as narrow bandwidth, low efficiency and gain. Many different solutions have been proposed, for instance: gain enhancement including: defected ground structure (DGS) [1], reflective surface [2], electromagnetic band gap (EBG) [3], bandwidth enhancement consisting of DGS [4], parasitic strips [5] and so on. One of the most attractive method to improve antenna parameters is DGS because of its ease in fabrication and low cost. DGS is an etched periodic or non-periodic cascaded configuration defect in ground of a planar transmission line, e.g., microstrip, coplanar and conductor backed coplanar wave guide [6]. By adjusting sizes of DGS, we can obtain the desired resonant frequency.

Besides, along with the rapid development of mobile communication technology, people's demand on high data rates and high reliability is becoming an urgent issue. There are two ways to increase the data transmission rate that is enhancing the channel bandwidth or increasing the number of transceiver antenna [7]. Multiple Input Multiple Output (MIMO) is a technology which allows increasing capacity and spectral efficiency through signal processing in both time and space domain. Currently, MIMO technology has been considered the best solution for most of the problems in wireless communication [8]. However, when antennas are placed close together, mutual coupling appears. Mutual coupling is the electromagnetic interaction between antenna elements in array. Mutual coupling decreases not the antenna efficiency, but also can change the radiation pattern of antenna as well. Therefore, mutual coupling is an urgent problem that needs to be solved. Many works have been conducted to improve parameters for antenna [9-11]. In [9], although the isolation between antenna elements is quite high $(30 \mathrm{~dB})$, gain of antenna is only under $6 \mathrm{dBi}$. In another document [10], although the bandwidth percentage of antenna is very high (96.2\%), mutual coupling of antenna is quite high (14 dB). In addition, the peak gain of antenna in this work is only $4 \mathrm{dBi}$. Similarly, the bandwidth percentage of antenna in [11] only achieves $5 \%$ (at central frequency of $5.8 \mathrm{GHz}$ ) although the isolation of antenna is acceptable (19 dB).

For this reason, this paper proposes a DGS to improve parameters for antenna. The proposed DGS is a compact and planar; therefore; it is easy for fabrication. The proposed structure is applied for a MIMO antenna including two sets of four elements $(2 \times 2)$. The antenna is yielded at the frequency of $5.5 \mathrm{GHz}$ for Wireless Local Area Network (WLAN) applications with the bandwidth of $500 \mathrm{MHz}$ corresponding to the bandwidth percentage of $9.1 \%$. In addition, the mutual coupling between elements is under $-20 \mathrm{~dB}$ while the gain and efficiency are $8.5 \mathrm{dBi}$ and over $90 \%$, respectively. The characteristics of the proposed antenna are demonstrated by Computer Simulation Technology (CST) software and validated by the measurements.

\section{Design of MiMO Array Antenna}

\subsection{The Proposed Defected Ground Structure}

Firstly, the model of the proposed DGS and its equivalent circuit are shown in Figure 1. The idea of the 

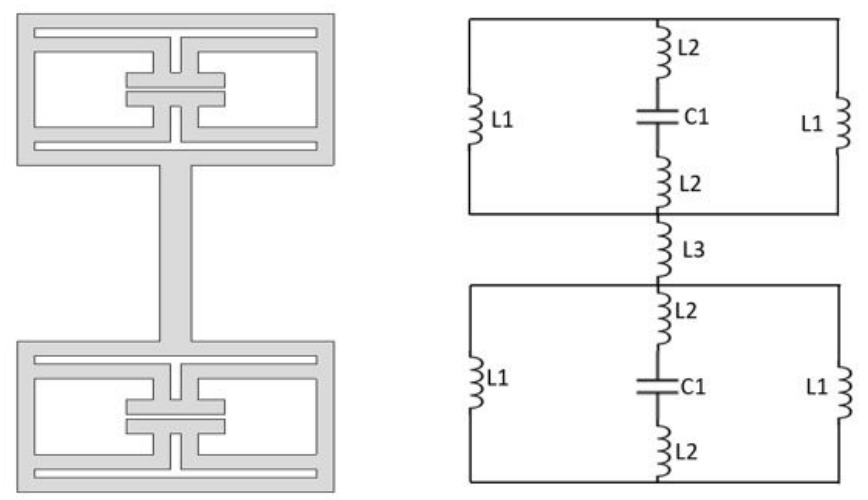

(a)
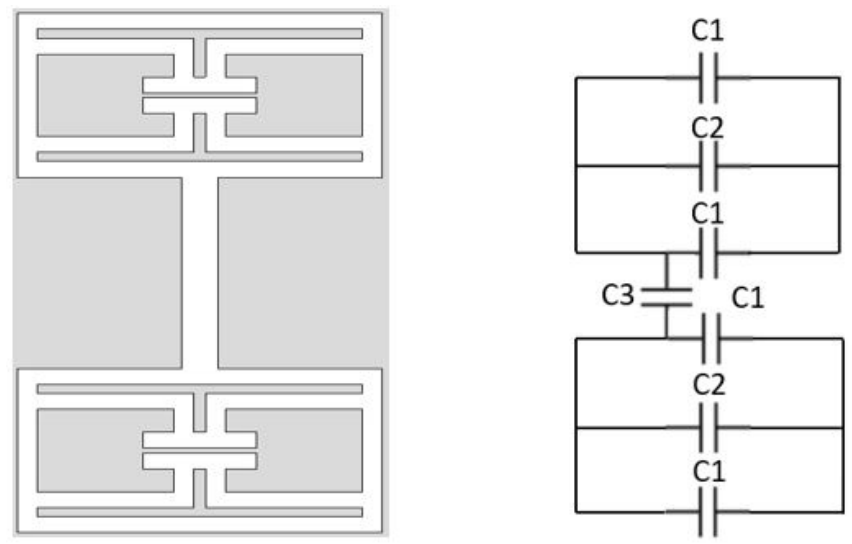

(b)

Figure 1. The model of the DGS: (a) the proposed structure; (b) the compensation structure.

proposed DGS is derived from the dumbbell-shaped DGS in [12]. By modifying the shape of structure, the paper has got a new structure. The proposed structure includes two rectangular shapes and they are connected to each other by a microstrip line. The proposed DGS is a planar structure, and it is, therefore, easy fabrication and low cost. To make a variety of parasitic capacitances (C) and inductances (L), the paper utilizes both the compensation structure and the proposed structure. While the compensation structure generates capacitances, the proposed structure produces inductances. This leads to an ease in optimization and achieves the better parameters for antenna.

\subsection{Design of Single Array}

Initially, a single array is designed for WLAN and its geometry is illustrated in Figure 2. It consists of four microstrip patch elements $(2 \times 2)$ connected together through power dividers placing on the dielectric substrate and the proposed DGS integrated on metal plane ground. Being on Rogers RT/Duroid ${ }^{\mathrm{TM}}$ 5880 substrate with thickness of $1.575 \mathrm{~mm}, \varepsilon r=2.2$ and $\tan \delta=0.0009$, the overall size of the proposed antenna is $72 \times 72 \times 1.575 \mathrm{~mm}^{3}$ while the distance between elements is approximately $\lambda_{0} / 2$, where $\lambda_{0}$ is the wavelength in free space at the frequency of 5.5 $\mathrm{GHz}$. Based on formulas in [13], the paper get a patch

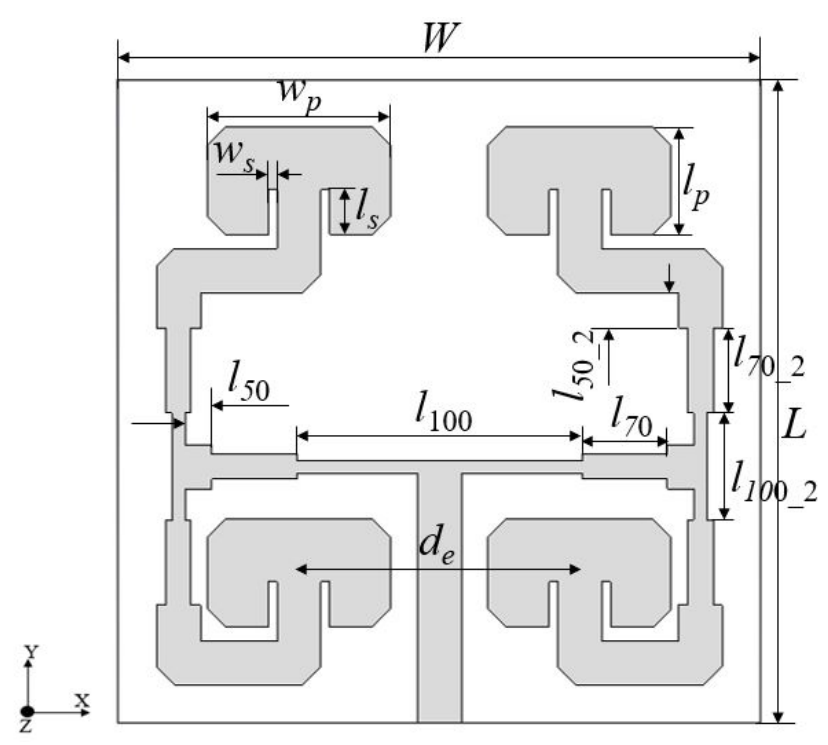

(a)

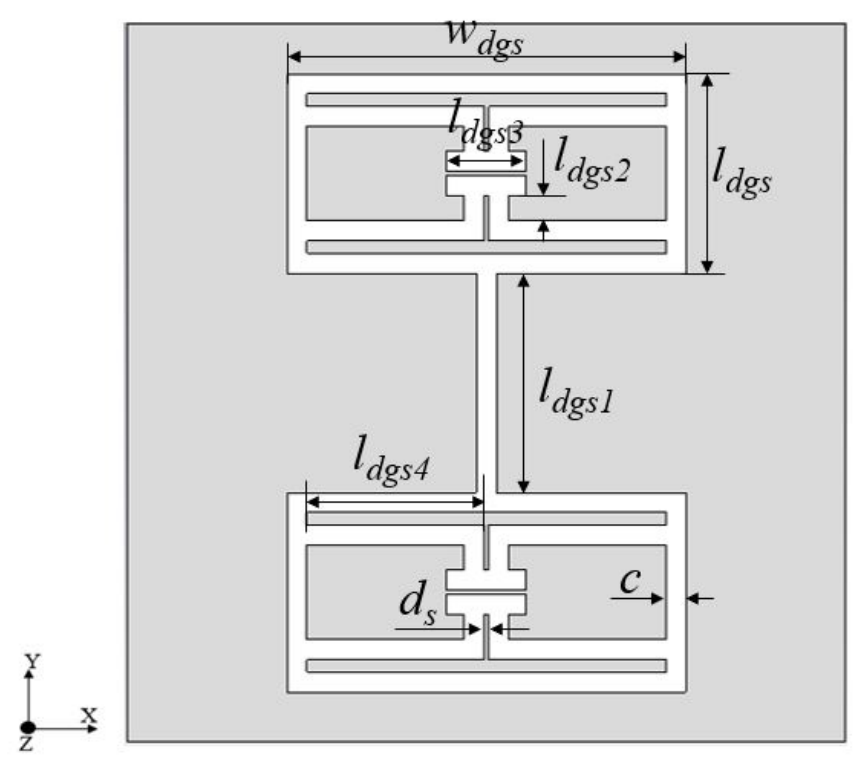

(b)

Figure 2. The model of the proposed array: (a) front view; (b) back view (dark colour for metal and light colour for substrate).

dimension of $20.5 \times 12 \mathrm{~mm}$. Meanwhile, the proposed DGS is integrated on the backside of the substrate layer.

The size of the DGS is $w_{d g s} \times l_{d g s}$ while the length of the microstrip line for connecting two rectangles is $l_{d g s 1}$. In addition, an indispensable component in antenna array is power dividers. To match impedance for them, transformers of $\lambda / 4$ are utilized, then, the impedance of transformers of $\lambda / 4$ is calculated as formula in [14]. These are equal power dividers, therefore, they are designed in order that the parameters of S21 and S31 are $-3 \mathrm{~dB}$. In other words, the power level at port 1 is twice times the one at port 2 and 3 . Table I shows some parameters of the proposed single array while Figure 3 presents S-parameters of the power divider. Here, the reflection coefficient at port 1 and power levels at port 2 and 3 of the power divider at the frequency of $5.5 \mathrm{GHz}$ are approximately $-28 \mathrm{~dB}$ and $-3.2 \mathrm{~dB}$, respectively. 
Table I

Some Parameters of the Proposed Single Array (Defined in Figure 2) of the Proposed Antenna Array

\begin{tabular}{|c|c|c|c|}
\hline Parameters & Value $(\mathrm{mm})$ & Parameters & Value $(\mathrm{mm})$ \\
\hline$W$ & 72 & $L$ & 72 \\
\hline$w_{p}$ & 20.5 & $l_{p}$ & 12 \\
\hline$w_{s}$ & 1 & $l_{s}$ & 5 \\
\hline$d_{e}$ & 27 & $w_{d g s}$ & 40 \\
\hline$l_{d g s 1}$ & 20 & $c$ & 4 \\
\hline$l_{100}$ & 29 & $l_{50}$ & 4.5 \\
\hline$l_{70}$ & 9.3 & $l_{100 \_2}$ & 14 \\
\hline$l_{50 \_2}$ & 3 & $l_{70 \_2}$ & 9.3 \\
\hline$d_{s}$ & 1 & $l_{d g s 2}$ & 2.5 \\
\hline$l_{d g s 3}$ & 10 & $w_{d g s 4}$ & 13.75 \\
\hline
\end{tabular}

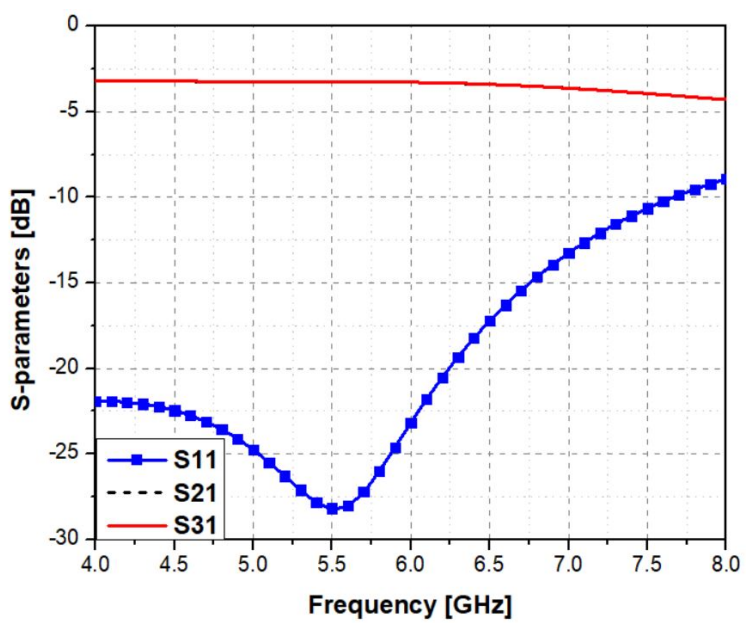

Figure 3. The parameters of the power divider.

\subsection{Design of MIMO Array Antenna}

The model of the MIMO array antenna is shown in Figure 4. The proposed antenna consists of two symmetrical sets of four elements $(2 \times 2)$ on the top of the substrate. The distance between radiation patches in the MIMO antenna is approximately $\lambda / 2$ while the closest gap from edge to edge of the two arrays is 6.5 $\mathrm{mm}$. The antenna is realized on Roger $5880^{\mathrm{TM}}$ substrate with the dimension of $140 \times 76 \times 1.575 \mathrm{~mm}^{3}$.

In order to enhance isolation for MIMO antenna, the DGS is integrated into the ground plane. The ground plane includes 3 cells of DGS with the distance from center to center between them of $42.5 \mathrm{~mm}$. Here, the size of the DGS is $32 \times 57 \mathrm{~mm}$. By adjusting some parameters such as $w_{d g s 2}, l_{d g s 2}, l_{d g s 3}, d_{d g s}, l_{c u t}, w_{c u t}$, we can obtain the desired resonant frequency. Table II shows some parameters of the MIMO array antenna.

\section{Results ANd Discussions}

\section{A. Simulation Results}

\subsection{Single array antenna}

Figure 5 shows the reflection coefficient of the single array. From Figure 5, we can see that the return loss of
Table II

Some Parameters of the MiMO Array Antenna

\begin{tabular}{|c|c|c|c|}
\hline Parameters & Value $(\mathrm{mm})$ & Parameters & Value $(\mathrm{mm})$ \\
\hline$W$ & 72 & $\mathrm{~L}$ & 140 \\
\hline$d_{a}$ & 5.05 & $w_{d g s 2}$ & 32 \\
\hline$l_{d g s 2}$ & 18 & $l_{d g s 3}$ & 21 \\
\hline$d_{d g s}$ & 42.5 & $w_{\text {cut }}$ & 35 \\
\hline$l_{\text {cut }}$ & 59 & & \\
\hline
\end{tabular}

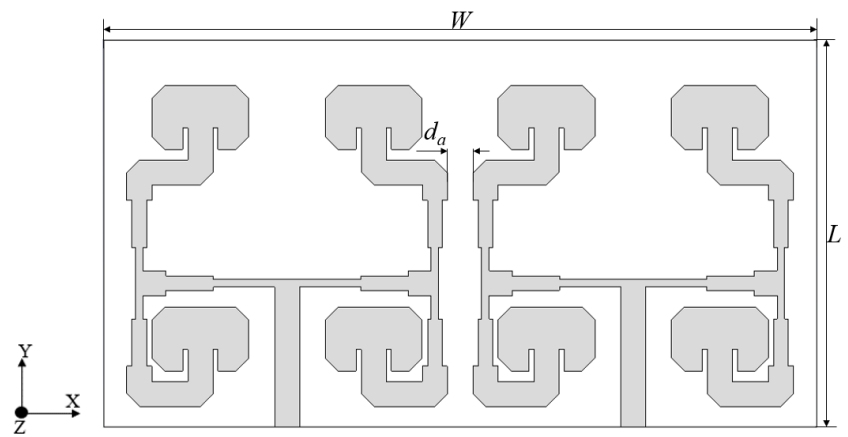

(a)

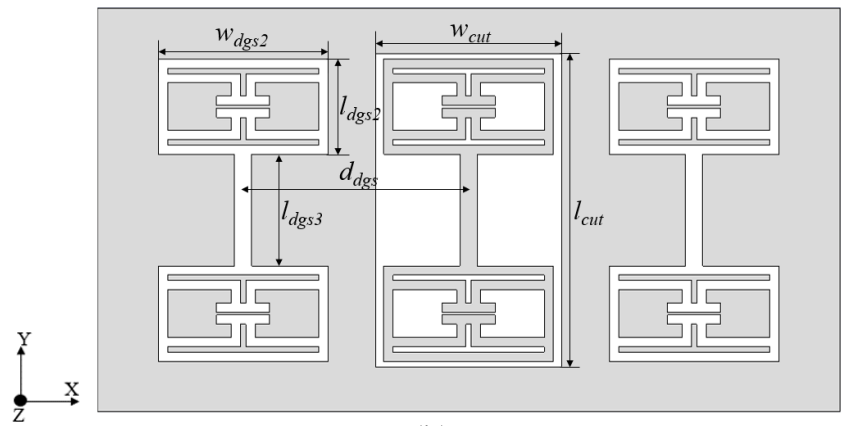

(b)

Figure 4. The model of the MIMO array antenna: (a) top view; (b) bottom view.

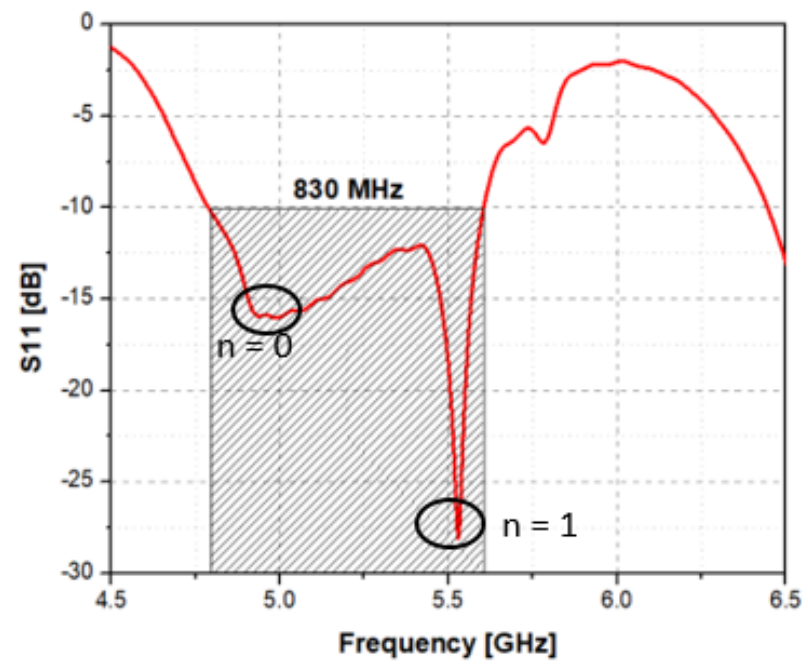

Figure 5. The reflection coefficient of the single array.

the antenna at the frequency of $5.5 \mathrm{GHz}$ is $-28 \mathrm{~dB}$ while the bandwidth is $830 \mathrm{MHz}$. In this case, the bandwidth is extended by making at least two consecutive resonant modes. It is clear that use of DGS on ground plane 


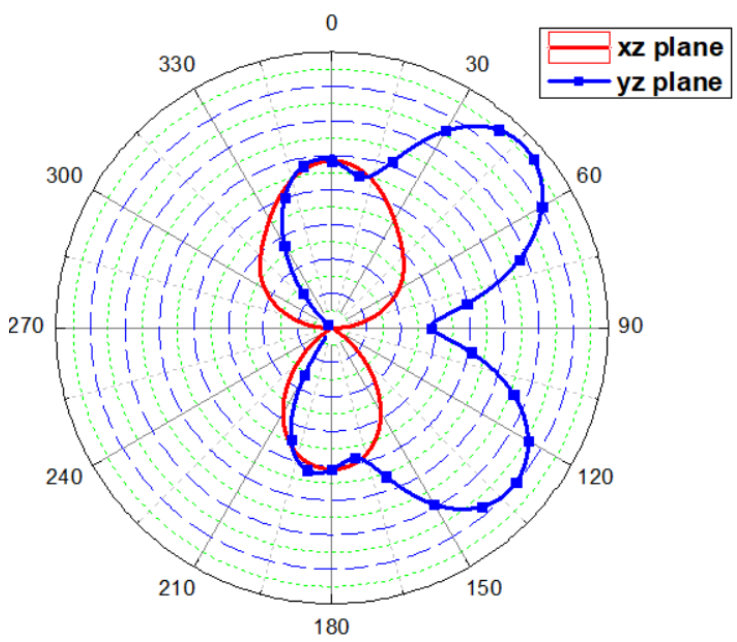

Figure 6. The $x z$ and $y z$ planes of the proposed array antenna.

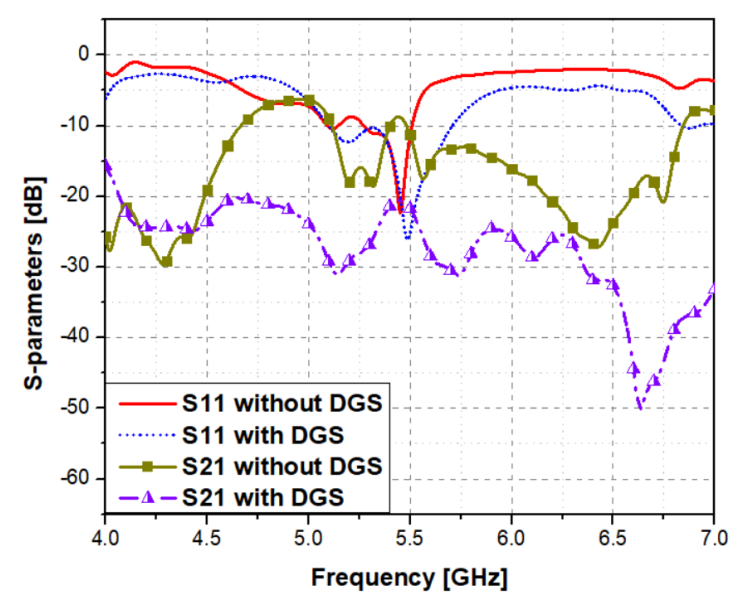

Figure 7. The simulated results of S-parameters with and without DGS.

made consecutive cavity resonators and this leads to creating resonant modes. Utilizing DGS not only enhances bandwidth for antenna, but also keep efficiency at high level. Here, the gain and efficiency of the antenna reach $7.3 \mathrm{dBi}$ and $87 \%$, respectively.

Figure 6 illustrates the $x z$ and $y z$ planes of the proposed. The antenna has the directivity of $7.8 \mathrm{dBi}$ while the angular width $(3 \mathrm{~dB})$ is 40.5 degree.

\subsection{MIMO Array Antenna}

As mentioned above, the goal for using DGS in MIMO array antenna is to reduce mutual coupling. This characteristic is illustrated in Figure 7, which displays a comparison of S-parameters in two cases of without and with the DGS.

As shown Figure 7, the isolation of antenna without DGS is only $10 \mathrm{~dB}$ while with case of DGS, this value is greater than $20 \mathrm{~dB}$ although the distance between elements is $30 \mathrm{~mm}$ (the distance between elements with DGS is $27 \mathrm{~mm}$ ). It is clear that there is a significant improvement in mutual coupling between antenna elements when DGS is used. This can be explained as following: The use of DGS causes a disturbance in current distribution [15] and this leads to current re-

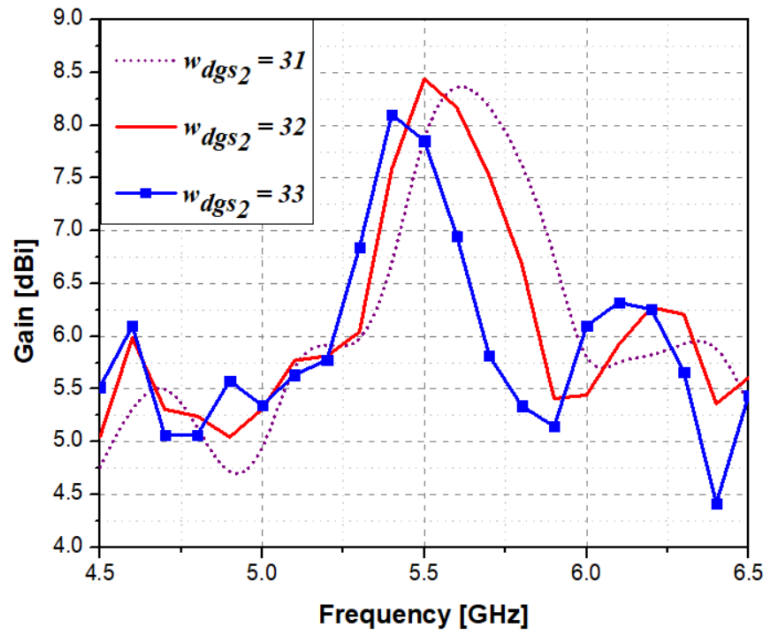

(a)

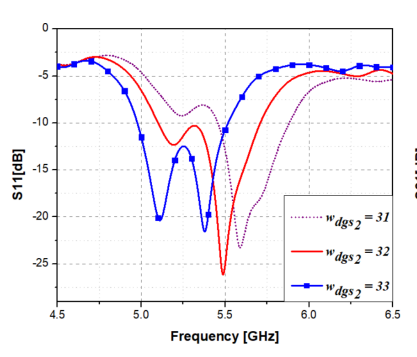

(b)

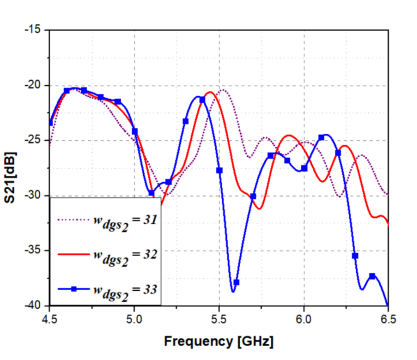

(c)
Figure 8. Simulated gain, S11 and S21 for the different widths of DGS.

disposal of antenna. The principle of gain enhancement as well as mutual coupling reduction, the author is presented more detailed in [16]. Then, we can adjust this distribution by changing the dimensions of DGS. As a result, the most currents are concentrated an identified place while the other places are limited. Therefore, the isolation of antenna is enhanced. In addition, by making parasitic inductances and capacitances, the size of an element is also reduced when DGS is used (the dimensions of an element are $19.5 \times 19.5 \mathrm{~mm}$ without DGS and $19 \times 12.5 \mathrm{~mm}$ with DGS). This shows that using DGS not only enhances isolation for antenna, but also reduces size for antenna. However, there is always a tradeoff in techniques which are used for improving parameters of antenna. In this case, utilizing DGS changed the position of the main lobe. Normally, if the place of the main lobe is at 0 degree, the main lobe place is 18 degree. However, this is acceptable.

Using DGS not only reduces mutual coupling, but also enhance bandwidth for antenna and this is also illustrated in Figure 7. If the bandwidth at $-10 \mathrm{~dB}$ of antenna $620 \mathrm{MHz}$ with DGS this data is only 240 $\mathrm{MHz}$ without DGS. Here, there are at least two created resonant modes and as a result, the bandwidth of antenna is improved, Moreover, the efficiency of antenna is remained at high level with $90 \%$.

Figure 8 illustrates simulated gain, S11 and S21 for the different widths of DGS ( $w_{\text {dgs } 2}$ in Table II). Although the S21 values are guaranteed under $-20 \mathrm{~dB}$ in three cases, gain and S11 achieve the best values with $w_{d g s 2}=32$. 


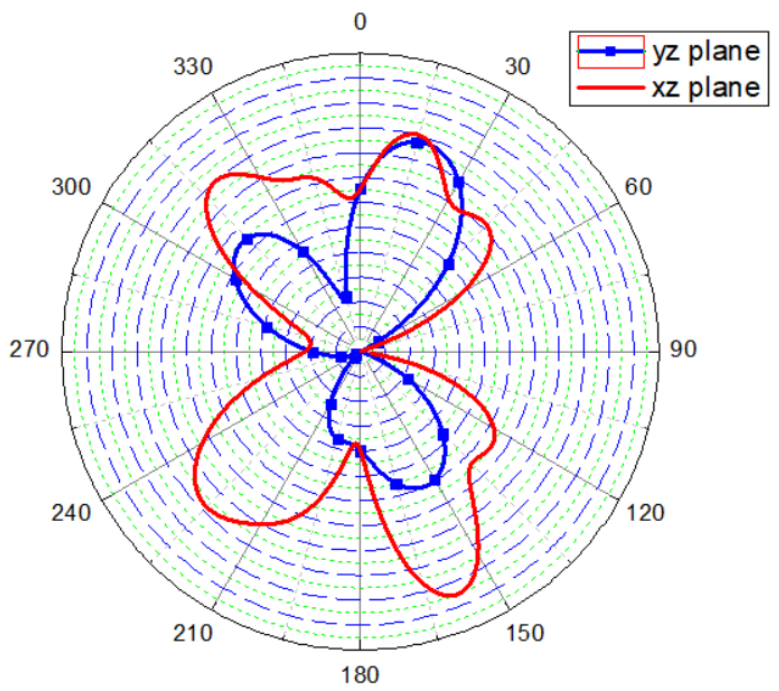

Figure 9. The xz and yz planes of the proposed MIMO array antenna.

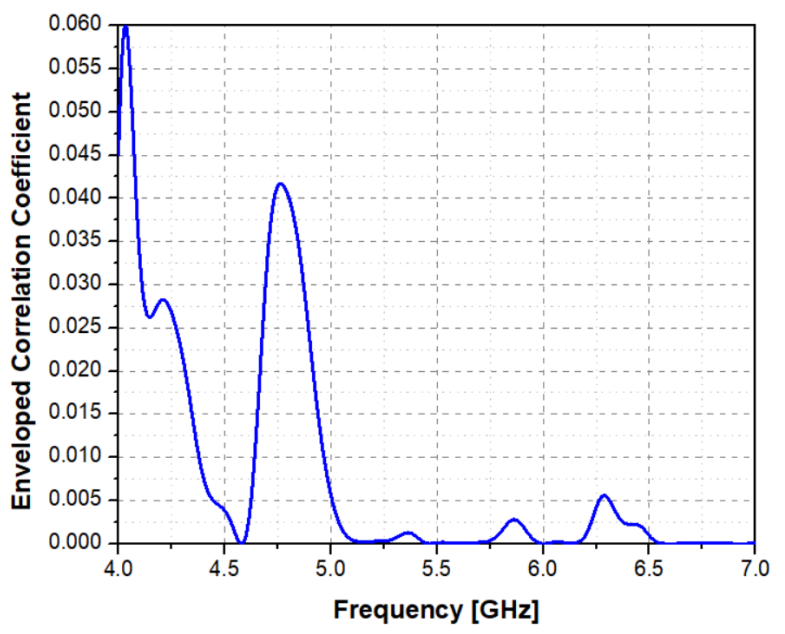

Figure 10. The ECC of the proposed array antennas.

Figure 9 shows the $x z$ and $y z$ planes of the proposed MIMO array antenna. The gain of the proposed array antenna gets $8.5 \mathrm{dBi}$ while the angular width $(3 \mathrm{~dB})$ is 37.4 degree. Besides, another important parameter in MIMO system to determine diversity performance is the envelope correlation coefficient (ECC). Here, ECC is defined as follows [17]:

$$
\rho_{e}=\frac{\left|S_{11}^{*} S_{12}+S_{21}^{*} S_{22}\right|^{2}}{\left(1-\left|S_{11}\right|^{2}-\left|S_{21}\right|^{2}\right)\left(1-\left|S_{22}\right|^{2}-\left|S_{12}\right|^{2}\right)} \text {. }
$$

Figure 10 and Figure 11 display the ECC and current distribution of the proposed antenna. From Figure 10 we can see that the ECC of the antenna is very small in a wide frequency range (under 0.0025 from $5.15 \mathrm{GHz}$ to $5.8 \mathrm{GHz}$ ). This shows that the isolation of the proposed antenna is quite high. Move to Figure 11, there are some places that the energy flows are concentrated higher other places (red color).

\section{B. Measurement Results}

For verification, the prototypes of the MIMO array antenna, as shown in Figure 12, are fabricated on
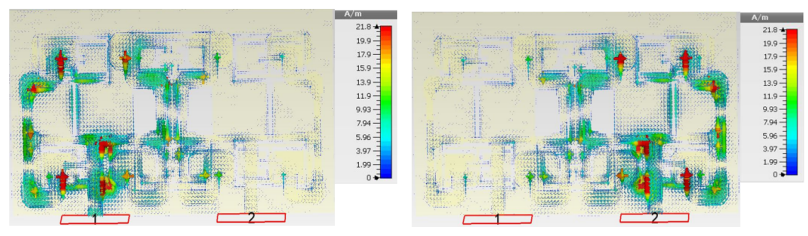

(a)

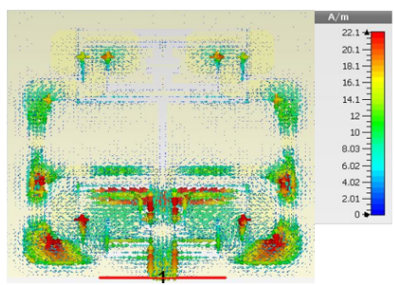

(b)

Figure 11. Current distribution of the proposed antenna: (a) MIMO antenna including $1^{\text {st }}$ element (left) and $2^{\text {nd }}$ element (right); (b) single antenna.
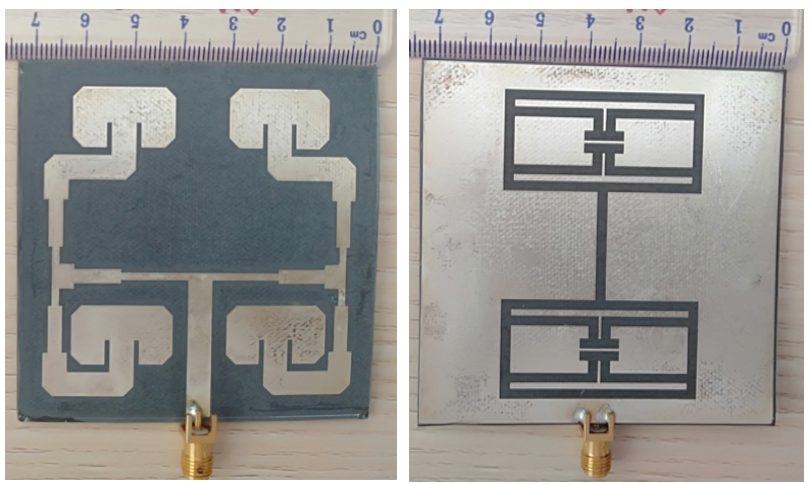

(a)
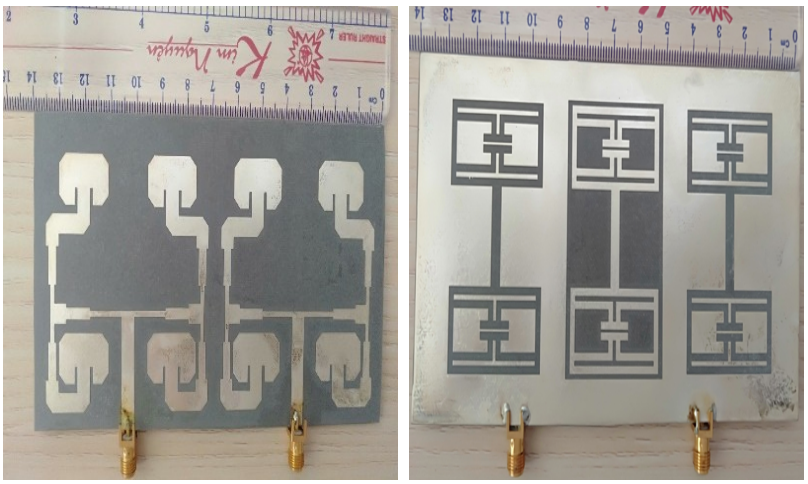

(b)

Figure 12. The prototypes of the proposed MIMO antenna: (a) single array antenna; (b) MIMO array antenna.

Rogers RT/Duroid ${ }^{\mathrm{TM}} 5880$ substrate with thickness of $1.575 \mathrm{~mm}, \varepsilon r=2.2$ and $\tan \delta=0.0009$. The overall sizes of the fabricated single and MIMO antennas are $72 \times 72 \times 1.575 \mathrm{~mm}^{3}$ and $140 \times 76 \times 1.575 \mathrm{~mm}^{3}$, respectively. The measured and the CST computed results for the fabricated MIMO and single array are given in Figure 13.

As displayed in Figure 13(a), the measured impedance bandwidth for $|S 11|<-10 \mathrm{~dB}$ is from $5.19 \mathrm{GHz}$ to $5.6 \mathrm{GHz}$ corresponding the bandwidth in percentage of approximately $7.4 \%$. Switch to Figure 13(b), the bandwidth of the MIMO antenna is 
Table III

The Comparison between the Previous Works and My Work

\begin{tabular}{|l|c|c|c|c|c|}
\hline References & {$[18]$} & {$[19]$} & {$[20]$} & {$[21]$} & My work \\
\hline Frequency [GHz] & 5.4 & 5.8 & $28 / 38$ & 5.8 & $\mathbf{5 . 5}$ \\
\hline Bandwidth [\%] & 38 & 4.7 & $14.3 / 5.26$ & 7.7 & $\mathbf{9 . 1}$ \\
\hline Isolation [dB] & 19 & 22 & 20 & 34 & $\mathbf{2 0}$ \\
\hline Efficiency [\%] & $\mathrm{x}$ & $\mathrm{x}$ & 73 & 53.7 & $\mathbf{9 0}$ \\
\hline Gain [dBi] & $\mathrm{x}$ & $\mathrm{x}$ & 7.5 & 5.3 & $\mathbf{8 . 2}$ \\
\hline Size & $1.98 \lambda \times 0.972 \lambda$ & $\mathrm{x}$ & $2.4 \lambda \times 1.8 \lambda$ & $1.87 \lambda \times 0.54 \lambda$ & $\mathbf{2 . 5 6} \lambda \times \mathbf{1 . 3 9} \lambda$ \\
\hline
\end{tabular}

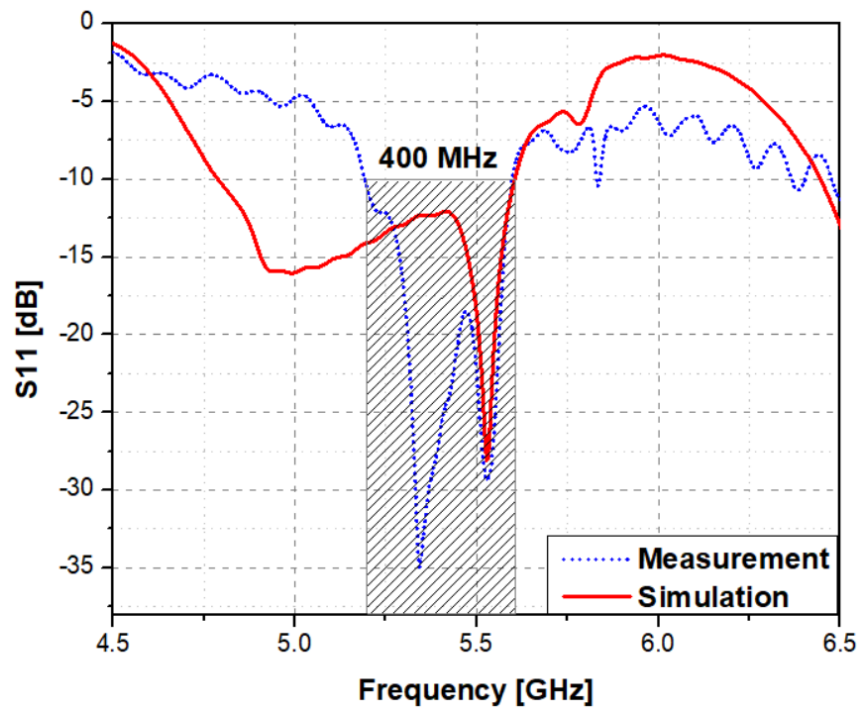

(a)

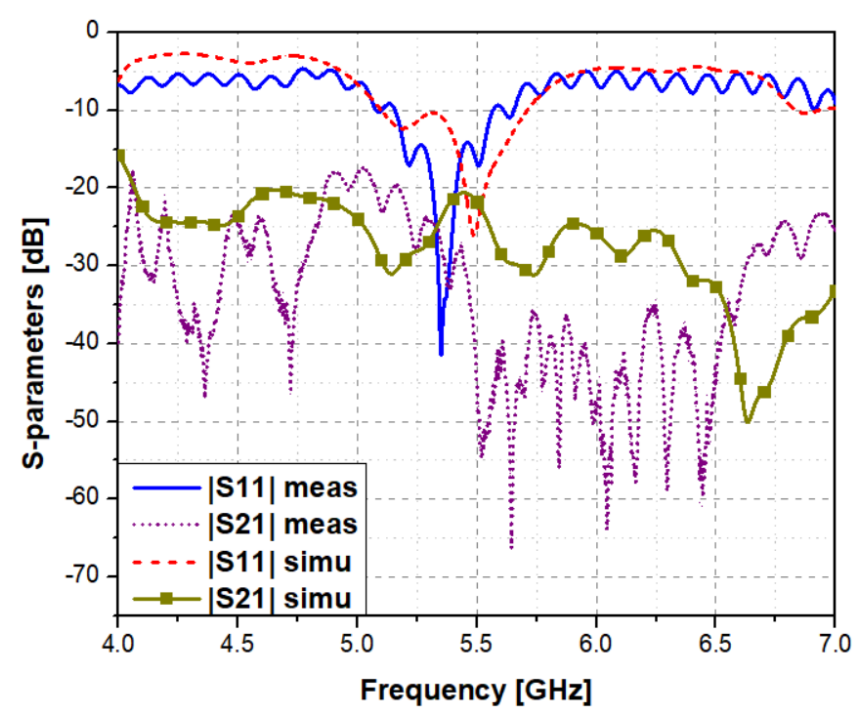

(b)

Figure 13. Measured results of the S-parameters: (a) single array antenna; (b) MIMO array antenna.

$500 \mathrm{MHz}(9.1 \%$, from 5.16 to $5.66 \mathrm{GHz})$. In addition, the mutual coupling of the antenna is under $-20 \mathrm{~dB}$ over a wide frequency range. In these cases, there are differences between measured and simulated results. This difference can be attributed to the tolerances of the fabricated antenna array. In addition, the SMA

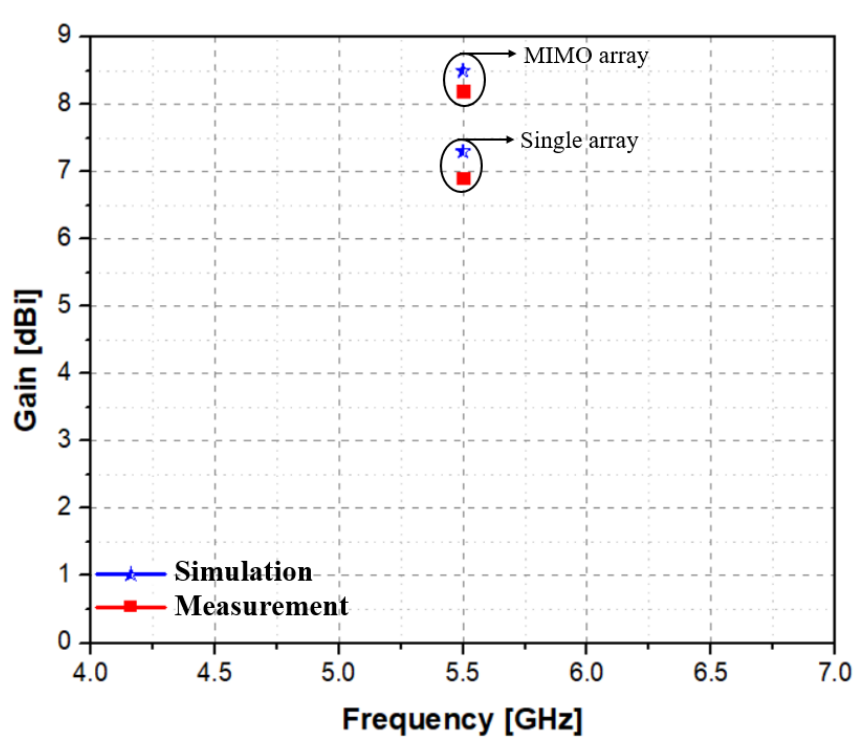

Figure 14. The measured gain of the proposed antenna.

soldering can cause an impedance variation of antenna and this directly affects to impedance matching. As a result, with MIMO antenna, there are a shift in frequency (S11) and the significant change between simulated and measured S21. However, there is a better result in measurement with single antenna when the second resonant mode is very close the simulated result and as a result, this mode is also the resonant frequency of antenna. Therefore, the frequency band for operating of the antenna is still ensured and this result is acceptable.

Figure 14 illustrates the measured results of gain of the proposed antenna. While the simulated results of the single array and MIMO array are $7.3 \mathrm{dBi}$ and $8.5 \mathrm{dBi}$, the measured ones for these figures are 7 $\mathrm{dBi}$ and $8.2 \mathrm{dBi}$, respectively. The gain values of antennas in measurement are lower than the figure in simulation. This cause may be due to insertion loss of SMA connectors. However, the difference is very small. The results in this work have also been compared with the previous works as shown in Table III. From Table III, we can see that although the isolation of antenna [19] is quite high with of $22 \mathrm{~dB}$, however, the bandwidth percentage is not high (under 5\%). In addition, the parameters of efficiency and gain did not show in these documents $[18,19]$. This is similar 
in [20] when the percentage of bandwidth is only 5.26. Besides, the efficiency and gain of antenna in [20] are not high $(73 \%$ and $7.5 \mathrm{dBi})$ although the antenna is yielded at frequencies of 28 and $38 \mathrm{GHz}$. In another studying [21], the gain and efficiency are very low (5.3 $\mathrm{dBi}$ and $53.7 \%$ ) although the mutual coupling between elements in antenna very low $(-34 \mathrm{~dB})$. Moreover, there is a narrow percentage of bandwidth in [21] $(7.7 \%)$. With document [18], the parameters are quite good (the bandwidth of percentage: $38 \%$ and the isolation: $19 \mathrm{~dB}$ ).

\section{Conclusion}

In this paper, a MIMO array antenna including two sets of four elements $(2 \times 2)$ and the proposed DGS for WLAN applications is investigated. The prototype, with an overall dimension of $140 \times 72 \times 1.575 \mathrm{~mm}^{3}$, yielded a measured bandwidth of 5.16-5.66 GHz (at -10 $\mathrm{dB})$. In addition, by using DGS integrated on ground plane, the antenna achieves a low mutual coupling (under $-20 \mathrm{~dB}$ ) in a wide frequency range. Moreover, the proposed antenna resulted in a peak gain of 8.2 $\mathrm{dBi}$ for measurement (8.5 $\mathrm{dBi}$ for simulation) and a total radiation efficiency of $90 \%$. With advantages consisting of low profile, easy fabrication with low cost, high isolation, wide bandwidth, and compact size, the proposed antenna is a quality candidate for using in wireless communication systems in practice.

\section{REFERENCES}

[1] M. S. Alam, M. T. Islam, and H. Arshad, "Gain enhancement of a multiband resonator using defected ground surface on epoxy woven glass material," The Scientific World Journal, vol. 2014, pp. 1-9, 2014.

[2] P. R. Prajapati and S. B. Khant, "Gain enhancement of UWB antenna using partially reflective surface," International Journal of Microwave and Wireless Technologies, vol. 10, no. 7, pp. 1-8, 2018.

[3] B. Mohamadzade and M. Afsahi, "Mutual coupling reduction and gain enhancement in patch array antenna using a planar compact electromagnetic bandgap structure," IET Microwaves, Antennas E Propagation, vol. 11, no. 12, pp. 1719-1725, 2017.

[4] A. Kandwal, R. Sharma, and S. Kumar Khah, "Bandwidth enhancement using Z-shaped defected ground structure for a microstrip antenna," Microwave and Optical Technology Letters, vol. 55, no. 10, pp. 2251-2254, 2013.

[5] H.-Y. Zhang, F.-S. Zhang, F. Zhang, T. Li, and C. Li, "Bandwidth enhancement of a horizontally polarized omnidirectional antenna by adding parasitic strips," IEEE Antennas and Wireless Propagation Letters, vol. 16, pp. 880-883, 2016.

[6] L. H. Weng, Y.-C. Guo, X.-W. Shi, and X.-Q. Chen, "An overview on defected ground structure," Progress In Electromagnetics Research B, vol. 7, pp. 173-189, 2008.

[7] W. Jiang, L. Yang, B. Wang, and S. Gong, "A high isolation dual-band MIMO antenna for WLAN application," in Proceedings of the International Symposium on Antennas and Propagation (ISAP). IEEE, 2017, pp. 1-2.

[8] A. J. Paulraj, D. A. Gore, R. U. Nabar, and H. Bolcskei, "An overview of MIMO communications-a key to gigabit wireless," Proceedings of the IEEE, vol. 92, no. 2, pp. 198218, 2004.

[9] J.-J. Liang, J.-S. Hong, J.-B. Zhao, and W. Wu, “Dual-band dual-polarized compact log-periodic dipole array for
MIMO WLAN applications," IEEE Antennas and Wireless Propagation Letters, vol. 14, pp. 751-754, 2014.

[10] R. Anitha, P. Vinesh, K. Prakash, P. Mohanan, and K. Vasudevan, "A compact quad element slotted ground wideband antenna for MIMO applications," IEEE Transactions on Antennas and Propagation, vol. 64, no. 10, pp. 4550-4553, 2016.

[11] N. Kumar and U. Kiran Kommuri, "MIMO antenna mutual coupling reduction for WLAN using spiro meander line UC-EBG," Progress In Electromagnetics Research C, vol. 80, pp. 65-77, 2018.

[12] M. S. Bhuiyan and N. C. Karmakar, "Defected ground structures for microwave applications," Wiley Encyclopedia of Electrical and Electronics Engineering, pp. 1-31, 1999.

[13] C. A. Balanis, Antenna theory: Analysis and design, 4th ed. John Wiley \& Sons, 2016.

[14] D. M. Pozar, Microwave engineering. John Wiley \& Sons, 2005.

[15] M. K. Khandelwal, B. K. Kanaujia, and S. Kumar, “Defected ground structure: fundamentals, analysis, and applications in modern wireless trends," International Journal of Antennas and Propagation, vol. 2017, pp. 1-22, 2017.

[16] N. N. Lan, "Gain Enhancement in MIMO Antennas Using Defected Ground Structure," Progress in Electromagnetics Research M, vol. 87, pp. 127-136, 2019.

[17] S. Blanch, J. Romeu, and I. Corbella, "Exact representation of antenna system diversity performance from input parameter description," Electronics Letters, vol. 39, no. 9, pp. 705-707, 2003.

[18] M. Y. Talha, K. J. Babu, and R. W. Aldhaheri, "Design of a compact MIMO antenna system with reduced mutual coupling," International Journal of Microwave and Wireless Technologies, vol. 8, no. 1, pp. 117-124, 2016.

[19] A. R. Mallahzadeh, S. Es' haghi, and A. Alipour, "Design of an E-shaped MIMO antenna using IWO algorithm for wireless application at $5.8 \mathrm{GHz}$," Progress In Electromagnetics Research, vol. 90, pp. 187-203, 2009.

[20] D. T. T. Tu, N. G. Thang, N. T. Ngoc, N. T. B. Phuong, and V. Van Yem, "28/38 GHz dual-band MIMO antenna with low mutual coupling using novel round patch EBG cell for 5G applications," in Proceedings of the International Conference on Advanced Technologies for Communications (ATC). IEEE, 2017, pp. 64-69.

[21] L. Malviya, R. K. Panigrahi, and M. V. Kartikeyan, “Circularly polarized $2 \times 2$ MIMO antenna for WLAN applications," Progress In Electromagnetics Research, vol. 66, pp. 97-107, 2016.

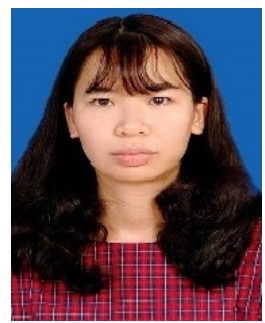

Nguyen Ngoc Lan received the Master and Ph.D. degrees in School of Electronics and Telecommunications, Hanoi University of Science and Technology, Vietnam, in 2014 and 2019 , respectively. Currently, she is a lecturer at the Faculty of Electronics and Telecommunications, Saigon University, Vietnam. Her research interests include microstrip antenna, mutual coupling, MIMO antennas, array antennas, reconfigurable antennas, polarization antennas, metamaterial, and metasurface. 